\title{
Editorial commentary on the Indian Journal of Gastroenterology May-June 2020
}

\author{
Jimmy K Limdi ${ }^{1,2}$ (D)
}

Published online: 24 July 2020

(C) Indian Society of Gastroenterology 2020

\section{"Learn from yesterday, live for today and hope for tomorrow. And never stop questioning." -Albert Einstein}

The emergence of the Severe Acute Respiratory Syndrome Corona Virus-2 (SARS-COV 2) from Wuhan, China in December 2019 and the resulting Corona Virus Disease (COVID-19) has gripped the world in a global pandemic from its unprecedented spread, changing our perceptions and paradigms around disease and the world, as we have known it. As of 11 July 2020, there have been 12,322,395 confirmed cases of COVID-19, including 556,335 deaths, reported to WHO [1] with 22,123 deaths in India [2]. Even as the world remains under the influence of this cataclysmic crisis, the medical and scientific communities have continued to work tirelessly and collaboratively to find answers for this hitherto unknown disease. Although a predominantly respiratory disease with multi-systemic ramifications, gastrointestinal (GI) symptoms such as diarrhea, vomiting, abdominal pain and hepatic abnormalities have been reported in up to $20 \%$ of patients with COVID-19, including those with minimal symptoms [3, 4]. The potential for SARS-COV2 to affect the GI tract and implications to patients with underlying GI and liver disease are of particular relevance to gastroenterologists. With this in mind, this issue focusses on COVID-19 with a series of research articles and clinical reviews on COVID-19 and GI disease, constituting essential and timely reading for the practising gastroenterologist. We hope you find these useful. And we wish you and your patients well...

Jimmy K Limdi

Jimmy.Limdi@nhs.net

1 Inflammatory Bowel Diseases, Division of Gastroenterology, The Pennine Acute Hospitals NHS Trust, Manchester M8 5RB, UK

2 Manchester Academic Health Sciences, University of Manchester, Manchester M8 5RB, UK

\section{Voluntary perioperative colorectal cancer registry from Kerala - an initial overview}

Colorectal cancer (CRC) ranks among the six most common malignancies in India; yet, outcomes data outside of selected centers are lacking [5]. Krishnan and colleagues report the first outcomes data from 1018 CRC cases from 15/25 participating centers of the Association of Surgical Gastroenterologists of Kerala CRC registry between 2016 and 2018 [6]. The majority of CRC cases were rectal $(39.88 \%)$ and rectosigmoid (20.33\%), with minimally invasive surgery (MIS) performed in $73 \%$ cases and $56.74 \%$ of colonic malignancies. MIS was associated with reduced length of hospital-stay compared to the open approach $(10.46 \pm 5.08$ vs. $12.26 \pm 6.03$ days; $p=0.001$ and $10.29 \pm 4.58$ vs. $12.46 \pm 6.014$ days; $p=<0.001$ ), with an overall $2.16 \%$ mortality. The data are limited by selection bias from voluntary participation but underpin the need for wider adoption, identifying outcome modifiers, and providing much-needed transparency for physicians, patients, and stakeholders in driving excellence in the overall delivery of care.

\section{Platelet to lymphocyte ratio as a predictive biomarker of liver fibrosis (on elastography) in patients with hepatitis C virus (HCV) -related liver disease}

Chronic hepatitis $\mathrm{C}$ with antecedent complications from cirrhosis is a major source of morbidity and mortality globally. Assessment of liver fibrosis is key to critical decisions with anti-viral therapy [7]. Liver biopsy remains the standard criterion for the histological evaluation although noninvasive assessment (transient elastography) is preferred for the serial evaluation but limited by cost and availability [7]. There is a resurgent interest in platelet to lymphocyte (PLR) and neutrophil to lymphocyte ratios (NLR) in predicting liver fibrosis beyond an assessment of inflammatory activity. 
Catanzaro and colleagues studied treatment-naive patients with chronic $\mathrm{HCV}$, who underwent clinical and laboratory assessments and transient elastography and were classified into Metavir F0-F4 [8]. Patients with F4 fibrosis (cirrhosis) had a lower PLR than the non-F4 group. Patients with PLR $>89$ demonstrated an increased risk of F4 fibrosis. No differences were noted in NLR values for both groups. Further studies in well-characterized cohorts incorporating liver biopsy are now needed to validate these findings.

\section{Decreasing major surgical rates for Crohn's disease in an emerging economy over two decades but is it due to biologic therapy?}

Biological therapies have re-defined our perceptions around meaningful disease control. "Treating to target" to achieve mucosal healing and deep remission early in the course of the disease may limit intestinal injury and ensuing disability [9]. Have biological therapies influenced a reduction in surgical resection rates?

Chuah and colleagues conducted a retrospective study across two tertiary centers in Malaysia and compared surgical resection rates in Crohn's disease (CD) patients in the prebiologic (cohort 1; 1991-2000) and immediate post-biologic era (cohort 2; 2001-2010) [10]. There was a significant reduction in the 7-year cumulative intestinal surgical rates between cohorts 1 and 2, from $21.4 \%$ to $10.2 \%(p=0.028)$, but there was no statistically significant difference in biologic exposure between those who underwent surgery and those who did not. A small sample size, variable disease duration, and timing of biologics may have influenced results. Long-term prospective studies with early use of optimized biological treatment are urgently needed [11].

\section{Gastrointestinal and hepatic manifestations of COVID-19 and their relationship to severe clinical course: A systematic review and meta-analysis}

Although predominantly a respiratory disease, GI manifestations are observed in COVID-19 [3]. Kumar and colleagues performed a systematic review and meta-analysis of 62 studies, to study the frequency of GI and hepatic manifestations and determine whether GI or hepatic manifestations are associated with a severe clinical course with COVID-19 [12]. Diarrhea was the most common GI symptom (9\%), followed by nausea/vomiting (5\%), abdominal pain (4\%), and chronic liver disease in $3 \%$ of patients.

A severe clinical course was seen in $20 \%$ with age $\geq 60$ years and underlying chronic comorbidity, but not chronic liver disease strongly associated with a severe clinical course.
Presence of diarrhea, high aspartate aminotransferase (AST), alanine aminotransferase (ALT), and bilirubin (odds ratio, [OR] 2), high prothrombin time (PT) (OR 4) and low albumin (OR 5) were associated with a severe clinical course. Patients with underlying GI disorders may be particularly vulnerable, and these findings and rapidly evolving evidence will influence our paradigms around COVID-19 and GI and liver disease.

\section{Poor outcomes in patients with cirrhosis and COVID-19}

The association between SARS-COV 2 infection and high mortality rates in people with cardiovascular and metabolic co-morbidities is well-recognized. Data on outcomes of SARS-COV 2 infection with chronic liver disease are scarce. Shalimar and colleagues from All India Institute of Medical Sciences (AIIMS), New Delhi report outcomes data from 28 patients with SARS-COV 2 infection and underlying chronic liver disease compared to age, sex, and severity-matched cirrhotic controls [13]. Twenty-six of 28 patients had cirrhosis, and one each had non-alcoholic fatty liver disease and extrahepatic portal venous obstruction. Mortality was numerically higher in COVID-19 (42.3\% vs. $23.1 \%, p=0.077$ ) and $100 \%$ among COVID-19 patients with acute-on-chronic liver failure, compared to $53.3 \%$ among controls $(p=0.015)$. Over $50 \%$ of patients had pneumonia, and on multivariate analysis, mechanical ventilation was independently associated with mortality (hazard ratio [HR] 13.680, $[p=0.025]$ ). These outcomes are similar to data from larger cohorts (SECURECIRRHOSIS registry) [14], which also reported 33\% mortality in COVID-19 patients with cirrhosis affirming our suspicions that patients with cirrhosis contracting SARS-COV 2 have poor outcomes.

\section{Compliance with Ethical Standards}

Conflict of Interest JKL declares that he has no conflict of interest.

Disclaimer The author is solely responsible for the contents of this paper. In no way, the Honorary Editor-in-Chief, Editorial Board Members, or the printer/publishers are responsible for the results/findings and content of this article.

\section{References}

1. https://covid19.who.int. accessed 11 July 2020.

2. https://www.mohfw.gov.in. accessed 11 July 2020.

3. Gu J, Han B, Wang J. COVID-19: gastrointestinal manifestations and potential fecal-oral transmission. Gastroenterology. 2020;158: 1518-9.

4. Guan WJ, Ni ZY, Hu Y, et al. Clinical characteristics of coronavirus disease 2019 in China. N Engl J Med. 2020;382:1708-20. 
5. Global Burden of Disease Cancer Collaboration, Fitzmaurice C, Akinyemiju TF, et al. Global, regional, and national cancer incidence, mortality, years of life lost, years lived with disability, and disability-adjusted life-years for 29 cancer groups, 1990 to 2016: a systematic analysis for the global burden of disease study. JAMA Oncol. 2018;4:1553-68.

6. Krishnan P, Kurumboor P, Varma D, et al. Voluntary perioperative colorectal cancer registry from Kerala - an initial overview. Indian J Gastroenterol. 2020;39. https://doi.org/10.1007/s12664-01900998-9.

7. EASL Recommendations on Treatment of Hepatitis C 2018. J Hepatol. 2018;69:461-511.

8. Catanzaro R, Sciuto M, Lanzafame C, et al. Platelet to lymphocyte ratio as a predictive biomarker of liver fibrosis (on elastography) in patients with hepatitis $\mathrm{C}$ virus (HCV)-related liver disease. Indian $\mathrm{J}$ Gastroenterol. 2020;39: https://doi.org/10.1007/s12664-02001038-7.

9. Limdi JK, Picco M, Farraye FA. A review of endoscopic scoring systems and their importance in a treat-to-target approach in inflammatory bowel disease (with videos). Gastrointest Endosc. 2020;91: $733-45$.
10. Chuah KH, Ali RAR, Hilmi IN. Decreasing major surgical rates for Crohn's disease in an emerging economy over two decades: is it due to biologic therapy? Indian J Gastroenterol 2020; 39: https:// doi.org/10.1007/s12664-020-01044-9.

11. Ungaro RC, Yzet C, Bossuyt $\mathrm{P}$, et al. Deep remission at 1 year prevents progression of early Crohn's disease. Gastroenterology. 2020;S0016-5085(20)30390-5.

12. Kumar A, Arora A, Sharma P, et al. Gastrointestinal and hepatic manifestations of Corona Virus Disease-19 and their relationship to severe clinical course: a systematic review and meta-analysis. Indian J Gastroenterol. 2020;39. https://doi.org/10.1007/s12664020-01058-3.

13. Shalimar, Elhence A, Vaishnav M, et al. Poor Outcomes in Patients with Cirrhosis and COVID-19. Indian J Gastroenterol. 2020; 39: https://doi.org/10.1007/s12664-020-01074-3.

14. https://covidcirrhosis.web.unc.edu/updates-and-data/ acessed 10 July 2020.

Publisher's note Springer Nature remains neutral with regard to jurisdictional claims in published maps and institutional affiliations. 